\title{
A Novel Inverse Kinematics Method for Upper-Limb Exoskeleton under Joint Coordination Constraints
}

\author{
Stefano Dalla Gasperina ${ }^{1}$, Keya Ghonasgi ${ }^{2}$, Ana C. de Oliveira ${ }^{2}$, \\ Marta Gandolla ${ }^{1}$, Alessandra Pedrocchi ${ }^{1}$ and Ashish Deshpande ${ }^{2}$.
}

\begin{abstract}
In this study, we address the inverse kinematics problem for an upper-limb exoskeleton by presenting a novel method that guarantees the satisfaction of joint-space constraints, and solves closed-chain mechanisms in a serial robot configuration. Starting from the conventional differential kinematics method based on the inversion of the Jacobian matrix, we describe and test two improved algorithms based on the Projected-Gradient method, that take into account jointspace equality constraints. We use the Harmony exoskeleton as a platform to demonstrate the method. Specifically, we address the joint constraints that the robot maintains in order to match anatomical shoulder movement and the closed-chain mechanisms used for the robot's joint control. Results show good performances of the proposed algorithms, which are confirmed by the ability of the robot to follow the desired taskspace trajectory while ensuring the fulfilment of joint-space constraints, with a maximum error of about 0.05 degrees.
\end{abstract}

\section{INTRODUCTION}

Humans have the remarkable ability to plan and perform motion in both the task space ("Where is my hand?"), as well as the joint space ("How much should I bend my elbow?") [1]. The ability to switch between these two planning strategies allows humans to perform a variety of complex motions towards the accomplishment of a wide array of functional goals. At the same time, thanks to the intrinsic kinematic redundancy of the arm, humans are able to overcome physical constraints by re-configuring the position of the arm. Robots have been developed with the aim of accomplishing similar functional tasks, but with higher levels of accuracy and repeatability. Joint space control is the most common approach in controlling robots, with single motor coupled to each degree of freedom. Inverse kinematics methods help bridge the gap between motor control and end-effector motion by transforming the motion from the task space to the joint space [2]. This conversion allows for seamless control of robots in the task space. Unfortunately, traditional inverse kinematics approaches are still unable to solve complex constraints in the task space and joint space simultaneously [3]. This need for complex simultaneous control is most apparent when robots are designed to cooperate with human users, as in the case of wearable exoskeletons.

The Harmony exoskeleton [4] (Fig. 1) is an upper body rehabilitation robot, and is thus required to maintain a

\footnotetext{
1 S. Dalla Gasperina, M. Gandolla and A. Pedrocchi are with NearLab and with the Department of Electronics, Information and Bioengineering, Politecnico di Milano, Italy.

2 K. Ghonasgi, A. de Oliveira and A. Deshpande are with ReNeu Lab and with the Department of Mechanical Engineering, University of Texas at Austin, TX, USA.
}

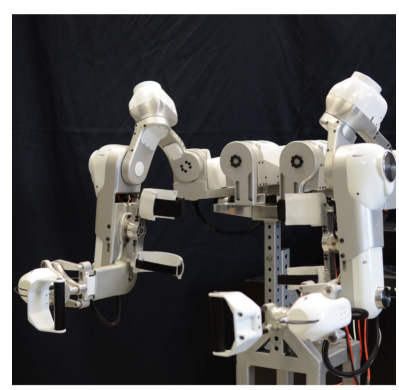

(a) Physical prototype

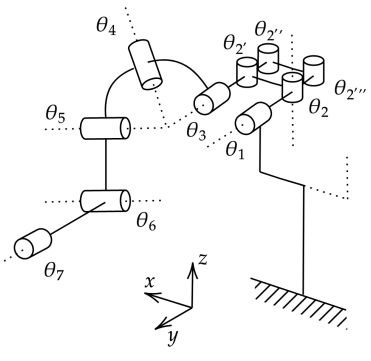

(b) Kinematic model.
Fig. 1: The Harmony exoskeleton [4] is a bi-manual upper body exoskeleton with 7 degrees of freedom in each arm.

high level of coordination with the wearer. An important characteristic of the robot is its ability to maintain healthy coordinated shoulder motion as described by the Scapulohumeral Rhythm (SHR) [4], [5]. However, maintaining this coordination becomes non-trivial when the goal of the motion is a functional task. As the robot uses parallelograms and virtual joints to ensure that the robot remains out of the wearer's way, standard methods of inverse kinematics adopted for serial manipulators are not directly applicable [6], [7]. The Harmony exoskeleton thus offers two challenges, 1) Joint-coordination constraints, and 2) Complex closed-chain internal mechanisms, that take the robot beyond the application of traditional inverse kinematics methods for serial manipulators. Being over-actuated in the task space (7 degrees of freedom in each arm), the Harmony exoskeleton benefits from the existence of a large set of possible solutions, referred to as the kinematic null space [8], which may be exploited based on a variety of performance metrics. Kinematic redundancy has been studied in literature for applications such as multi-level minimization [9] and redundant manipulator control similar to the Harmony exoskeleton [10]. However, the redundancy in manipulator task space has not been exploited to simultaneously solve for multiple cost functions under conflicting dynamic and kinematic constraints. We address this gap in the current work by presenting and validating a set of novel methods that exploit the kinematic null space to solve inverse kinematics problems under joint-coordination constraints.

\section{BACKGROUND}

\section{A. The Inverse Kinematic Approach}

In this section, we briefly introduce conventional inverse kinematics to motivate the need for novelty. In particular, 
one of the most common approaches relies on differential kinematics, which gives the relationship between the joint velocities and the end-effector linear and angular velocities. In particular, such a mapping is described by the Jacobian matrix, which depends on the robot's configuration and is given in the well-known form:

$$
\dot{\boldsymbol{x}}=\boldsymbol{J}(\boldsymbol{\theta}) \dot{\boldsymbol{\theta}}
$$

where $\dot{\boldsymbol{x}}$ is an $m \times 1$ vector representing three linear and three rotational velocities of the end-effector; $\dot{\boldsymbol{\theta}}$ is an $n \times 1$ vector consisting of the joint velocities; and $\boldsymbol{J}(\boldsymbol{\theta})$ is an $m \times n$ Jacobian matrix. When dealing with kinematically redundant robots $m<n$, thus the Jacobian matrix becomes a nonsquare matrix and the right pseudo-inverse solution is needed to solve the inverse problem of (1):

$$
\dot{\boldsymbol{\theta}}=\boldsymbol{J}^{\dagger} \dot{\boldsymbol{x}}
$$

where $\boldsymbol{J}^{\dagger}$ is the Moore-Penrose right pseudo-inverse. This approach gives the best possible solution that minimizes the norm of the joint velocities, but is still unstable near singularities. Thus, in certain configurations, the robot is rendered unable to move the end-effector in a certain way. This is because the Jacobian matrix becomes ill-conditioned, and the pseudo-inverse method results in very large joint velocities that tend to explode. The damped least squares method, introduced by Nakamura et al. [11], can be used to avoid such instability by adding a damping term $\lambda$ to the solution of Equation 1:

$$
\boldsymbol{J}^{\dagger \lambda}=\boldsymbol{J}^{T}\left(\boldsymbol{J} \boldsymbol{J}^{T}+\lambda \boldsymbol{I}\right)^{-1}
$$

Several variations of this approach have been proposed in literature in past decades [12]-[14]. However, these approaches do not explicitly consider additional constraints to the inverse kinematics problem. The human arm, and consequently any arm exoskeleton, such as Harmony, is characterized by a kinematic redundancy that can be exploited to fulfill additional requirements. Fortunately, the solution of the inverse kinematics problem for highly redundant robots permits the existence of a subspace $\boldsymbol{N}(\boldsymbol{J})$ of joint velocities, called the Null-space, characterized by joint velocities that do not produce any end-effector movement, at the given configuration. To exploit this subspace of internal motions, the previously defined solution (2), called the particular solution, can be extended to include an additional term, called the homogeneous solution. This term explores the Null-space to find a solution that satisfies additional arbitrary constraints, expressed in joint velocities, and can be formalized as:

$$
\dot{\boldsymbol{\theta}}=\boldsymbol{J}^{\dagger} \dot{x}+\boldsymbol{P} \dot{\boldsymbol{q}}
$$

where $\dot{\boldsymbol{q}}$ is the joint-space constraint expressed as a perturbation from the particular solution and $\boldsymbol{P}$ is a $n \times n$ matrix defined so that $\boldsymbol{J} \boldsymbol{P} \dot{\boldsymbol{q}}=0$, which means that the perturbation $\dot{\boldsymbol{q}}$ doesn't produce any movement of the end-effector in the task-space. Liegeois [15] presented one of the possible implementations of the $\boldsymbol{P}$ matrix, which has been termed the Null-space Projector, which permits the projection of the perturbation $\dot{\boldsymbol{q}}$ into the Null-space generating motions of the manipulator that do not change position or orientation of the end-effector. The overall solution becomes:

$$
\dot{\boldsymbol{\theta}}=\boldsymbol{J}^{\dagger} \dot{\boldsymbol{x}}+\left(\boldsymbol{I}_{\boldsymbol{N}}-\boldsymbol{J}^{\dagger} \boldsymbol{J}\right) \dot{\boldsymbol{q}}
$$

where $\boldsymbol{I}_{\boldsymbol{N}}$ is an $n \times n$ identity matrix.

In general, the choice of $\dot{\boldsymbol{q}}$ can be determined through a Projected-Gradient (PG) algorithm that defines it as:

$$
\dot{\boldsymbol{q}}=k \frac{\partial H(\boldsymbol{\theta})}{\partial \theta}
$$

where $H(\boldsymbol{\theta})$ is a differentiable objective function and $k>0$ is a weighting factor. Siciliano and colleagues [16] described different methods to define the optimal $H(\boldsymbol{\theta})$ objective function given the required constraints. Moreover, Liegeois presented a cost function that, by minimizing the distance of joint angles from the center of their ranges, maximizes the distance of joint variables from mechanical joint limits [15]. More recent literature also discusses multi-objective cartesian control [17], and in the context of redundant robots [9]. However, no objective function has been proposed for equality constraints in joint-space to guarantee coordinated motion among joints. In this paper, we approach the problem of managing the trade-off between task-space motion accuracy, and joint space constraint requirements under complex kinematic constraints by exploring the null subspace. Ultimately, we will present a set of methods that may be used given varying prioritization of performance criteria.

\section{B. Kinematic Model of the Harmony Exoskeleton}

We now introduce the platform used to validate the novel inverse kinematics method, and which further allows us to develop an idea regarding the importance of considering joint-space and kinematic constraints of robots in general. The Harmony exoskeleton (Fig. 1a) is a bi-manual upper body rehabilitation robot. It has seven degrees of freedom (DOF) in each arm (Fig. 1b), of which one joint controls wrist pronation-supination, one controls elbow flexionextension, and the remaining five are used to replicate the anatomical shoulder movement (the shoulder girdle). This is accomplished by combining a 3 DOF glenohumeral (GH) ball-and-socket joint and a 2 DOF rotation-translation joint for the GH joint. The 2 DOF mechanism allows the shoulder girdle to track the anatomical center of rotation (COR) of the $\mathrm{GH}$ joint. The methods used to maintain this coordination result in two contrasting constraints on the inverse kinematics of the robot.

1) Scapulohumeral Rhythm: Upper-arm movement is characterised by a coordinated motion of the shoulder. As the humerus of the upper-arm moves, so does the shoulder complex in such a way that a non-linear relationship is maintained between the humerothoracic elevation and the shoulder complex elevation. As a result, the center of rotation of the glenohumeral joint moves along with the subject, and must thus be followed accurately by the robot (see Fig. 2a and Fig. 2b). The nonlinear relationship, also known as the 


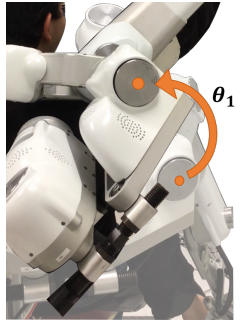

(a) Front view.

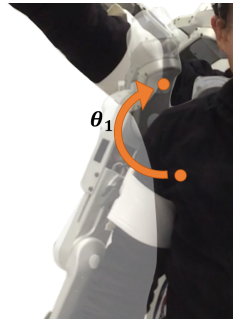

(b) Back view.

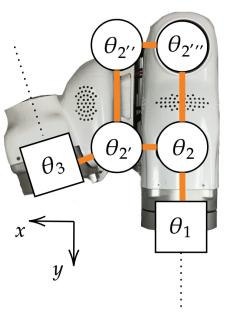

(c) Top View.
Fig. 2: (a) and (b) show the robot compensating for the movement of the subject's glenohumeral as the humerus is elevated. (c) shows the parallelogram mechanism from a top view.

Scapulohumeral Rhythm (SHR), is defined as [18], [19]:

$$
\theta_{1}=0.0036 \beta_{h}^{2}+0.085 \beta_{h}
$$

where, $\theta_{1}$ is the angle of rotation for the elevation of the shoulder and $\beta_{h}$ is the humeral elevation angle that can be calculated from the translation of the shoulder girdle mechanism. For more detail, refer to Kim and colleagues [18] where the authors designed and implemented a control strategy in order to maintain this complex coordination. As discussed in Section II, this kinematic coupling is not compatible with conventional inverse kinematics techniques, and thus cannot be used for task-space motion control.

2) Closed Chain Mechanism: In order to maintain the SHR coordination of the shoulder complex, the 2 DOF translation joint was designed as described in Figure 2c. This mechanism allows the shoulder ball-and-socket joint to be co-located with the center of rotation of the anatomical Glenohumeral joint during movement. In particular, the revolute joint $\theta_{1}$ performs shoulder elevation and depression and the parallelogram represented by $\theta_{2} \theta_{2}^{\prime} \theta_{2}^{\prime \prime} \theta_{2}^{\prime \prime \prime}$ performs shoulder protraction and retraction. Kinematically, the parallelogram has only one degree of freedom, and can be treated as a single joint. However, serial chain inverse kinematics methods cannot handle parallelogram joints without simplifying assumptions and modifications. To this end, the parallelogram structure can be split in two joints by adding a virtual joint (i.e. $\theta_{2}^{\prime}$ ) to the serial chain. The virtual joint must always match the following relationship to guarantee the kinematic compatibility:

$$
\theta_{2}^{\prime}=-\theta_{2}
$$

\section{METHODS}

In this section we first introduce a cost function to meet joint-coordination equality constraints, following which we propose two distinct inverse kinematics algorithms to be compared with the gold-standard differential kinematics algorithm. Quantitative metrics and qualitative results are computed respectively in simulation environment and with the robot to validate the feasibility and accuracy of the proposed algorithms.

For the sake of simplicity, as the robot is controlled in discrete time, from now on we will address the inverse kinematics problem considering finite differences as shown in (9):

$$
\Delta \boldsymbol{\theta}=\boldsymbol{J}^{\dagger} \Delta \boldsymbol{x}+P_{\boldsymbol{N}(\boldsymbol{J})} \Delta \boldsymbol{q}
$$

where $\Delta \boldsymbol{\theta}, \Delta \boldsymbol{x}$ and $\Delta \boldsymbol{q}$ represents respectively the differential value in discrete time of joint angles, cartesian position and perturbation angles.

\section{A. Objective function for joint-space equality constraints}

As mentioned in Section II-B, the kinematic model of the Harmony exoskeleton is characterized by two critical issues, that can be both included in the model as jointspace equality constraints. With reference to the previously described Projected-Gradient algorithm, we hereby propose a simple cost function that minimizes the distance between joint variables and target values, and is iteratively computed according to each specific constraint. The objective function can be written as:

$$
H(\boldsymbol{\theta})=\frac{1}{2} \sum_{i=1}^{N}\left(\theta_{i}-\bar{\theta}_{i}\right)^{2}
$$

where $N$ is the number of active joints, $\theta_{i}$ is the actual joint value and $\bar{\theta}_{i}$ is the target value imposed by the joint coordination of the robot. By computing the finite differences and differentiating the objective function, $\Delta q$ becomes:

$$
\Delta q_{i}=k\left(\theta_{i}-\bar{\theta}_{i}\right)
$$

This perturbation, $\Delta q_{i}$, tries to clamp the i-th joint angle to its target value $\bar{\theta}_{i}$, with a lower-priority compared to the task-space high-priority objective. In these experiments the weighting factor is fixed to $k=1$, so as to maximize the accuracy within the null-space. In the following discussion, we only consider the joint equality constraints due to the SHR and to the closed-chain parallelogram mechanism, as anticipated in Section II-B.

\section{B. Proposed Algorithms}

Given the highly redundant nature of the human arm, it is reasonable to expect the existence of a large nullspace for the realization of constraints both in task and joint space. Slotine and Siciliano [20] first introduced a method to deal with conflicting task-space constraints, each of them characterized by a Jacobian matrix. Baerlocher et al. [21] and other studies in the field of hierarchical inverse kinematics control [22] proposed methods to extend the problem to $n$ priority levels, but all are focused on the fulfillment of task-space problem and do not take into consideration jointcoordination constraints. For these reasons, such approaches are not suitable for our application, where the coordinated motion among the joints is more relevant with respect to the task-space position of the end-effector of the exoskeleton. Here, we restate the current gold-standard algorithm, followed by the two novel algorithms. Each of these is compared with the traditional approach, to solve the inverse kinematics problem of Harmony exoskeleton:

0) Jacobian Inverse Kinematics (J-IK)

The traditional inverse kinematics method exploits the right pseudo-inverse of the Jacobian to compute a configuration 


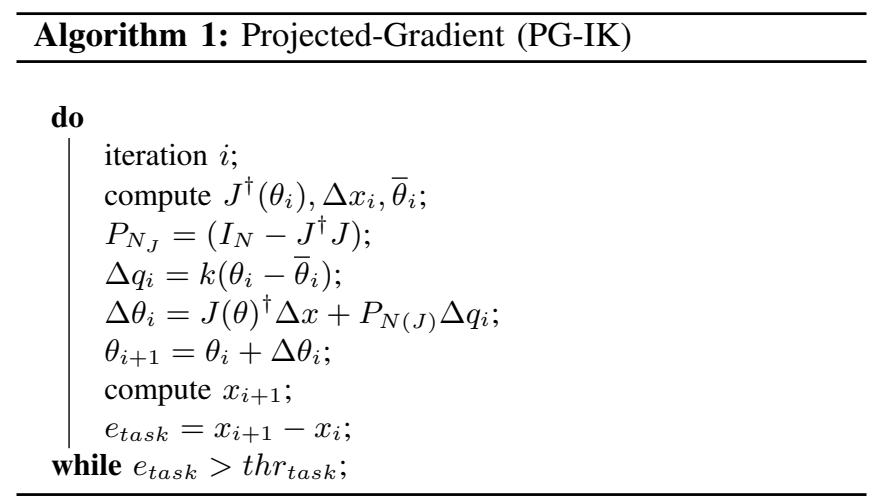

in the joint-space until the cartesian error between desired and computed positions is below a certain threshold. This approach has been widely discussed in literature [2].

1) Projected-Gradient (PG-IK):

The Algorithm 1, which can be considered an extension of the J-IK, is introduced to explore the null-space and introduce joint-space equality constraints. In particular, the objective function presented in Equation 10 allows the algorithm to compute the joint perturbation $(\Delta \boldsymbol{q})$ to be projected onto the null-space. By the definition of the PG method (Eq. 5), it should be noted that the constraints introduced by the PG-IK have a lower priority with respect to the primary kinematic constraint.

2) Constrained Projected-Gradient (CPG-IK):

We thus propose the Algorithm 2 that not only exploits the null-space to fulfill additional restrictions, but also adds an additional convergence condition. Specifically, in order to exit the convergence loop, the error in joint-space must be below a desired threshold $\left(t h r_{\text {joint }}=0.05\right.$ degrees), so as to guarantee a good coordination among joints.

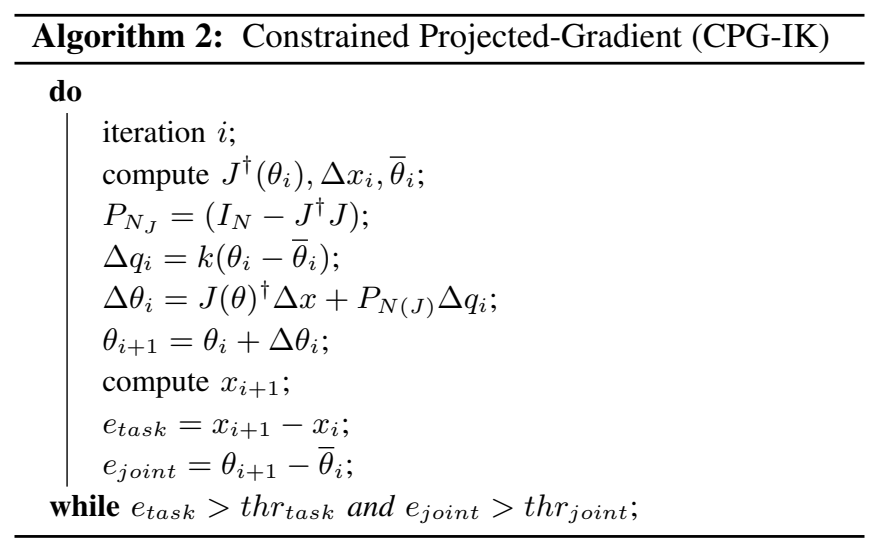

\section{EXPERIMENTAL DESIGN}

The kinematic model of the Harmony exoskeleton, described in Section II-B, has been used for the validation of the above mentioned algorithms. Two experimental methods have been performed: the first one considers the quantitative measurement of the performances of the algorithms in the simulation environment, while the second one qualitatively assesses the feasibility of the online application with the robot during more complex movements.

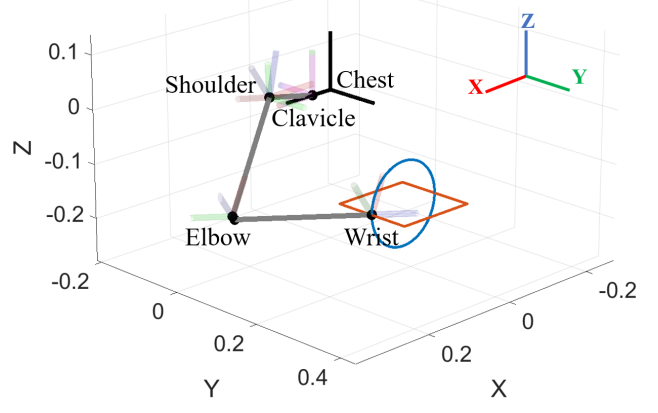

Fig. 3: Experimental trajectories: A square (red) and a circle (blue) trajectory are shown in the horizontal and frontal plane, respectively.

\section{A. Quantitative Performance Metrics}

To validate the performance of the algorithms, the inverse kinematics problem has been solved both for circular and square trajectories to be followed by the end-effector (i.e. the hand) of the exoskeleton. Each circular and squared trajectory has been performed in the frontal, sagittal and horizontal planes (Fig. 3), then the results were aggregated. Circular trajectories in task space have a diameter of 15 $\mathrm{cm}$, while square trajectories are characterized by sides of $15 \mathrm{~cm}$. Circular trajectories have been performed both at constant and variable speed, square trajectories are tested only at constant speed. In particular, when variable speed is considered, we divided the trajectory into intervals and randomly picked the samples within each interval, so as to render different speeds of movement.

The inverse kinematics problem has been solved offline in the MATLAB environment and several evaluation metrics have been computed as described below:

1) Number of iterations $\left(I_{\#}\right)$ : The average number of iterations needed to exit the convergence loop for each trajectory point. Results are presented in terms of median and interquartile values, along all the trajectories.

2) Task-space error $\left(E_{3 D}\right)$ : Euclidean distance between target and actual task-space positions. Results are presented in terms of maximum euclidean distance, expressed in millimeters $[\mathrm{mm}]$.

3) Joint-space error $\left(E_{\theta_{1}}, E_{\theta_{2}}\right)$ : Difference between desired and actual joint angles. In particular we evaluate how good the inverse kinematics method is to match joint-space equality constraints. We mainly consider the constraints related to $\theta_{1}$ (for the Scapulohumeral Rhythm) and to $\theta_{2}^{\prime}$ (for the closed-chain mechanism), as described in Section II-B. We present the maximum joint-space error expressed in degrees $\left[{ }^{\circ}\right]$.

4) Smoothness $\left(S_{M}\right)$ : Movement smoothness is computed as described in [23], where the metric is defined as the time integral of absolute value of jerk measures along the trajectory. Smoothness values are then sum among joint angles and expressed in arbitrary units.

The objective of these experiments is to provide a quantitative comparison of the performance of each algorithm, with the aim of identifying the one most suitable for the control of the exoskeleton's end-effector position. 
TABLE I: Performance Metrics Results

\begin{tabular}{cccccc}
\hline \multirow{2}{*}{ Algorithm } & \multicolumn{5}{c}{ Circular Shape - Constant Speed } \\
\cline { 2 - 6 } & $I_{\#}^{\mathrm{a}}$ & $E_{3 D}^{\mathrm{b}}$ & $E_{\theta_{1}}$ & $E_{\theta_{2}^{\prime}}$ & $S_{M}^{\mathrm{c}}$ \\
\hline J-IK & $1(0)$ & 0.0032 & 8.100 & 17.44 & 0.004 \\
PG-IK & $1(0)$ & 0.0034 & 0.207 & 0.387 & 0.015 \\
CPG-IK & $4(1)$ & 0.0027 & 0.049 & 0.050 & 1.060 \\
\hline \hline \multicolumn{6}{c}{ Circular Shape - Variable Speed } \\
\cline { 2 - 6 } & $I_{\#}$ & $E_{3 D}$ & $E_{\theta_{1}}$ & $E_{\theta_{2}^{\prime}}$ & $S_{M}$ \\
\hline J-IK & $1(0)$ & 0.0116 & 8.140 & 17.45 & 9.946 \\
PG-IK & $1(0)$ & 0.0107 & 0.254 & 0.494 & 10.58 \\
CPG-IK & $3(2)$ & 0.0072 & 0.050 & 0.049 & 15.03 \\
\hline \hline & \multicolumn{7}{c}{ Square Shape - Constant Speed } \\
\cline { 2 - 6 } & $I_{\#}$ & $E_{3 D}$ & $E_{\theta_{1}}$ & $E_{\theta_{2}^{\prime}}$ & $S_{M}$ \\
\hline J-IK & $1(0)$ & 0.0052 & 9.006 & 22.13 & 0.448 \\
PG-IK & $1(0)$ & 0.0063 & 0.235 & 0.453 & 0.555 \\
CPG-IK & $4(3)$ & 0.0001 & 0.035 & 0.050 & 1.827 \\
\hline
\end{tabular}

${ }^{a}$ Median number of iterations (Interquartile Range)

${ }^{\mathrm{b}}$ Max Euclidean error

${ }^{\mathrm{c}}$ Cumulative Smoothness along joints, in a.u.

\section{B. Qualitative online feasibility assessment}

Upon identifying the appropriate algorithm in terms of performance metrics, the feasibility of an online application was tested both on the Virtual Robot Experimentation Platform (V-REP) simulator and on the robot.

To validate the entire workspace of the robot and the range of joint speeds, we included more complex trajectories at different velocities of execution. This was done in the form of a spiral in the 3D space, and the drawing of a complex shape in the frontal plane. The joint profiles were computed offline in MATLAB, following which the simulator and the robot performed the same task-space motion. Given that the exoskeleton uses impedance control and not position control, the resulting motion was expected to follow the same overall pattern of the trajectory while allowing for deviations in the motion, depending on the commanded motor stiffness, due to the internal friction and weight of the robot. As a consequence the discussion with respect to the robot data is qualitative and not quantitative. The V-REP simulations naturally do not suffer from the same issue.

\section{RESUlts AND DisCUSSION}

\section{A. Quantitative Performance Metrics}

In this section the performances of the three algorithms J-IK, PG-IK, and CPG-IK, are compared for each trajectory. Since no significant changes were found among the same trajectory on different planes, we present the results obtained by aggregating the data of experiments in frontal, horizontal and sagittal planes. In Table I, performances for constant speed circular trajectory, randomized speed circular trajectory and constant speed square trajectory are reported.

It should be noted that, in all cases, relatively few iterations were needed to converge to a solution that satisfied the constraints and both PG-IK and CPG-IK were able to significantly improve the joint-space error $\left(E_{\theta}\right)$. Also, since

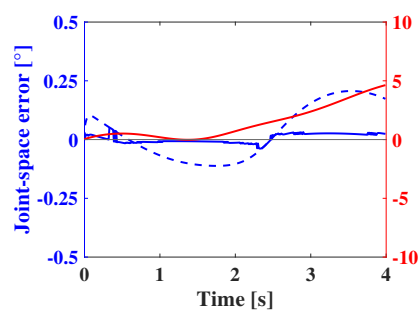

(a) SHR constraint $\left(\theta_{1}\right)$

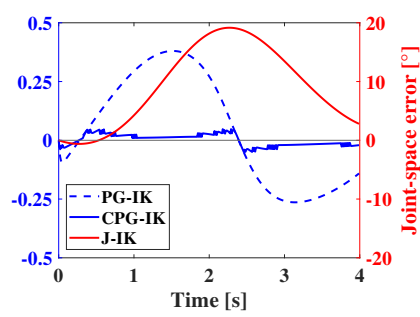

(b) Closed-chain constraint $\left(\theta_{2}^{\prime}\right)$
Fig. 4: The joint-space constraints error is minimized by the CPGIK algorithm, with respect to J-IK and PG-IK.

the CPG-IK required more iterations to find a solution, the task-space optimization problem was also able to benefit from the additional computations, leading to a slight decrease in the cartesian error. Moreover, it appears that the nature of the equality constraints, i.e. whether they were derived from a physiological human arm coordination or from a constraint in the kinematic model, did not influence the performance of the algorithms. Both the proposed algorithms were able to exploit the redundancy to meet either restriction.

If the proposed algorithms were able to guarantee joint coordination, as a consequence, the addition of joint-space constraints worsened the other performances (Table I). The effect is especially pronounced for the CPG-IK, indeed more repetitions were needed to compute a valid solution and the smoothness of movement was worsened. Fig. 4 shows the joint constraints errors during a circular trajectory in the taskspace, for both the shoulder-coordination constraint (a) and the closed-chain constraint (b). It can be noticed that the errors computed with J-IK are at least one order of magnitude higher with respect to PG-IK and CPG-IK. Also, the CPG-IK added some oscillations in the trajectory, leading to a worse smoothness metric.

\section{B. Qualitative online feasibility assessment}

A composite 2-dimensional motion was used to test the inverse kinematics algorithms in the robot. The same motion was performed in the V-REP environment as well as by the Harmony Exoskeleton. The motion was described to be planar in the frontal plane. A camera was used to help track the end effector position and visually compare the resulting motion to the desired one. The comparison shown in Fig. 5a demonstrates that the robot was able to match the commanded task space motion well, but with some error as a consequence of the robot's impedance control. Depending on the commanded speed and control stiffness of each motor, the resulting motion of the robot shows varying levels of deviation from the desired trajectory. In order to demonstrate the application of our method to cover 3-dimensional (3D) motion, we created an additional 3D composite trajectory, included in the supplementary video as a demonstration.

\section{Limitations}

The presented algorithms were tested only with the equality constraints introduced by the Harmony exoskeleton, while no constraints were put on joint limits or workspace 


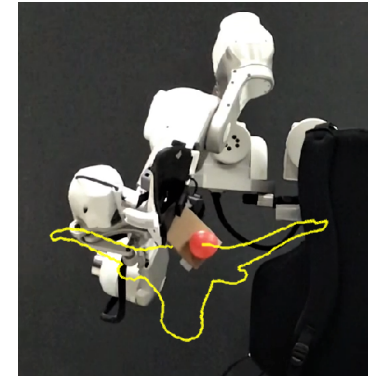

(a) Harmony exoskeleton

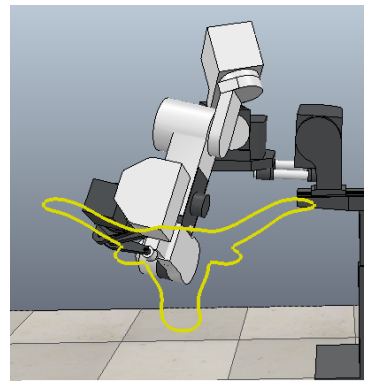

(b) V-REP Simulation
Fig. 5: Complex movements performed by the Harmony exoskeleton (a) and by the V-REP Simulation (b).

boundaries. Also, we believe that including additional constraints at the velocity level might improve the smoothness of the movement. Finally, considering the applicability of this approach on the Harmony exoskeleton, the accuracy of the motion could be improved by adding a closed-loop inverse kinematics logic within the real-time control of the exoskeleton, so as to compensate the task-space error along the desired trajectory.

\section{CONCLUSION}

In this work we presented and compared a set of novel methods to address the inverse kinematics problem for an upper-limb exoskeleton such as the Harmony exoskeleton. The kinematic model of the robot has been described and two distinct joint-space constraints, prevent the use of traditional inverse kinematics methods for serial manipulators, have been introduced into the inverse kinematics problem. The presented algorithms first apply the conventional differential kinematics to solve a task-space problem, then the corresponding null-space (afforded by the robot's kinematic redundancy) is explored to maintain joint-space constraints, as well as to ensure the kinematic integrity of the robot.

The Projected-Gradient (PG) and the Constrained Projected-Gradient (CPG) methods have been tested on different trajectories with the kinematic model of the exoskeleton. We saw that CPG-IK had the best performances in terms of joint-space errors, while PG-IK was slightly faster and did not compromise the smoothness of the movement. It can be concluded that, if a high-accuracy in joint-space coordination is required, the CPG-IK algorithm is ideally suited, while the PG-IK may be preferred if a trade-off among smoothness, computational demand and joint coordination is desired.

Finally, we showed that the Harmony exoskeleton was able to follow the desired task-space trajectory with good accuracy even when complex shapes were generated. Thus, the contribution of this study lies in the ability of the proposed methods to optimize for multiple objectives while adhering to conflicting kinematic and dynamic constraints. While the objectives and constraints presented in this work relate to joint coordination and end effector tracking, the methods presented here may be further extended. We therefore believe that this approach could be used for several applications, especially when robots are expected to interact with humans.

\section{REFERENCES}

[1] E. Burdet, D. W. Franklin, and T. E. Milner, Human robotics: neuromechanics and motor control. MIT press, 2013.

[2] S. R. Buss, "Introduction to inverse kinematics with jacobian transpose, pseudoinverse and damped least squares methods," IEEE Journal of Robotics and Automation, vol. 17, no. 1-19, p. 16, 2004.

[3] B. Siciliano, L. Sciavicco, L. Villani, and G. Oriolo, Robotics: modelling, planning and control. Springer Science \& Business Media, 2010.

[4] B. Kim and A. D. Deshpande, "An upper-body rehabilitation exoskeleton harmony with an anatomical shoulder mechanism: Design, modeling, control, and performance evaluation," The International Journal of Robotics Research, vol. 36, no. 4, pp. 414-435, 2017.

[5] J. Crosbie, S. L. Kilbreath, L. Hollmann, and S. York, "Scapulohumeral rhythm and associated spinal motion," Clinical biomechanics, vol. 23, no. 2, pp. 184-192, 2008.

[6] Y. Han, J. Wu, C. Liu, and Z. Xiong, "Static model analysis and identification for serial articulated manipulators," Robotics and ComputerIntegrated Manufacturing, vol. 57, pp. 155-165, 2019.

[7] L. Unzueta, M. Peinado, R. Boulic, and Á. Suescun, "Full-body performance animation with sequential inverse kinematics," Graphical models, vol. 70, no. 5, pp. 87-104, 2008.

[8] A. Dietrich, C. Ott, and A. Albu-Schäffer, "An overview of null space projections for redundant, torque-controlled robots," The International Journal of Robotics Research, vol. 34, no. 11, pp. 1385-1400, 2015.

[9] D. Chen, S. Li, W. Li, and Q. Wu, "A multi-level simultaneous minimization scheme applied to jerk-bounded redundant robot manipulators," IEEE Transactions on Automation Science and Engineering, vol. 17, no. 1, pp. 463-474, 2019.

[10] H. Sadeghian, L. Villani, M. Keshmiri, and B. Siciliano, "Task-space control of robot manipulators with null-space compliance," IEEE Transactions on Robotics, vol. 30, no. 2, pp. 493-506, 2013.

[11] Y. Nakamura and H. Hanafusa, "Inverse Kinematic Solutions With Singularity Robustness for Robot Manipulator Control," Journal of Dynamic Systems, Measurement, and Control, vol. 108, no. 3, pp. 163-171, sep 1986.

[12] S. Chiaverini, B. Siciliano, and O. Egeland, "Review of the damped least-squares inverse kinematics with experiments on an industrial robot manipulator," IEEE Transactions on control systems technology, vol. 2, no. 2, pp. 123-134, 1994.

[13] A. S. Deo and I. D. Walker, "Overview of damped least-squares methods for inverse kinematics of robot manipulators," Journal of Intelligent and Robotic Systems, vol. 14, no. 1, pp. 43-68, 1995.

[14] S. R. Buss and J.-S. Kim, "Selectively damped least squares for inverse kinematics," Journal of Graphics tools, vol. 10, no. 3, pp. 37-49, 2005.

[15] A. Liegeois et al., "Automatic supervisory control of the configuration and behavior of multibody mechanisms," IEEE transactions on systems, man, and cybernetics, vol. 7, no. 12, pp. 868-871, 1977.

[16] B. Siciliano, "Kinematic control of redundant robot manipulators: A tutorial," Journal of intelligent and robotic systems, vol. 3, no. 3, pp. 201-212, 1990.

[17] E. M. Hoffman, A. Laurenzi, L. Muratore, N. G. Tsagarakis, and D. G. Caldwell, "Multi-priority cartesian impedance control based on quadratic programming optimization," in 2018 IEEE International Conference on Robotics and Automation (ICRA). IEEE, 2018, pp. 309-315.

[18] B. Kim and A. D. Deshpande, "Controls for the shoulder mechanism of an upper-body exoskeleton for promoting scapulohumeral rhythm," in 2015 IEEE International Conference on Rehabilitation Robotics (ICORR). IEEE, 2015, pp. 538-542.

[19] N. Barnett, R. Duncan, and G. Johnson, "The measurement of three dimensional scapulohumeral kinematics-a study of reliability," Clinical Biomechanics, vol. 14, no. 4, pp. 287-290, 1999.

[20] S. B. Slotine, "A general framework for managing multiple tasks in highly redundant robotic systems," in proceeding of 5th International Conference on Advanced Robotics, vol. 2, 1991, pp. 1211-1216.

[21] P. Baerlocher and R. Boulic, "An inverse kinematics architecture enforcing an arbitrary number of strict priority levels," The visual computer, vol. 20, no. 6, pp. 402-417, 2004.

[22] M. Liu, Y. Tan, and V. Padois, "Generalized hierarchical control," Autonomous Robots, vol. 40, no. 1, pp. 17-31, 2016.

[23] D. Goldvasser, C. A. McGibbon, and D. E. Krebs, "High curvature and jerk analyses of arm ataxia," Biological cybernetics, vol. 84, no. 2, pp. 85-90, 2001. 